\title{
Quantum Recurrence Relation and Its Generating Functions
}

\author{
by
}

\author{
Noboru NAKANISHI
}

\begin{abstract}
A homogeneous linear recurrence relation with constant coefficients is extended to the case in which the relevant quantities are not necessarily commutative with each other. Two kinds of its generating functions are explicitly given, and a relation between them is established. This result is essentially nothing but the reduction to a common (symmetric) denominator for a sum of fractions of non-commutative quantities.
\end{abstract}

2010 Mathematics Subject Classification: Primary 00A99; Secondary 47S99.

Keywords: non-commutative quantities, recurrence relation, difference equation, generating function, reduction to a common denominator.

\section{$\S 1$. Introduction}

We consider a sequence $\left\{\Phi_{n}\right\}$ defined by the following homogeneous linear recurrence relation (or linear difference equation [1], [4]):

$$
\Phi_{n+N-1}=\sum_{k=0}^{N-1} a_{k} \Phi_{n+k-1} \quad(n \geq 1),
$$

where $a_{0}, a_{1}, \ldots, a_{N-1}$ and $\Phi_{0}, \Phi_{1}, \ldots, \Phi_{N-1}$ are given quantities independent of $n$.

Usually, these quantities are assumed to be mutually commutative as a matter of course. It is an interesting problem to see what happens if they are not necessarily commutative, that is, we wish to consider the "quantum" version of the recurrence relation. A simple example (with $N=2$ ) is the recurrence relation for the Bessel functions of the first, second and third kinds:

$$
Z_{n+1}(x)=-2 \frac{d}{d x} Z_{n}(x)+Z_{n-1}(x) .
$$

Communicated by M. Kashiwara, Received August 28, 2011.

N. Nakanishi: Professor Emeritus of RIMS, Kyoto University, Kyoto, 606-8502, Japan; e-mail: nbr-nak@trio.plala.or.jp

(C) 2013 Research Institute for Mathematical Sciences, Kyoto University. All rights reserved. 
In this example, the coefficient $a_{0}$ contains a differential operator with respect to a parameter $x$; hence the commutativity between $a_{0}$ and $\Phi_{n}$ no longer holds, but the coefficients $a_{j}$ remain mutually commutative. What we wish to consider is the case in which they are not necessarily mutually commutative.

In the present paper, we investigate the generating functions of the noncommutative extension of the sequence (1.1). Our main result is stated in Section 2. It is noteworthy that Gelfand et al. [2] proposed the theory of quasi-determinants, which is a non-commutative extension of determinants. In the course of our investigation, we encounter the same problem as the one discussed by them.

The present paper is organized as follows. In Section 2, two kinds of generating functions of a homogeneous linear recurrence sequence with non-commutative constant coefficients are defined and their explicit expressions are given. It is pointed out that they are related through the Laplace transform. This implies a certain identity for fractional expressions, which is the main goal of the present paper. In Section 3, the case of $N=2$ is explicitly worked out. In Section 4 , it is shown how to calculate the denominator and numerator functions appearing in the above identity. In Section 5, this method is applied to the case of $N=3$. The identity of Section 2 is proved in Section 6. The final section is devoted to a discussion in which it is pointed out that this identity can be regarded as the reduction to a common denominator for a sum of fractions of non-commutative quantities.

\section{$\S 2$. Generating functions}

We consider two kinds of generating functions [3] of the sequence $\left\{\Phi_{n}\right\}$ :

(a) ordinary generating function,

$$
g(t)=\sum_{n=0}^{\infty} \Phi_{n} t^{n}
$$

(b) exponential generating function,

$$
f(t)=\sum_{n=0}^{\infty} \Phi_{n} \frac{t^{n}}{n !}
$$

In the above, the infinite sums should be understood as formal power series.

Proposition 2.1. The ordinary generating function (2.1) is given by

$$
g(t)=\left(1-\sum_{k=1}^{N} a_{N-k} t^{k}\right)^{-1} \sum_{m=0}^{N-1}\left(1-\sum_{k=1}^{N-m-1} a_{N-k} t^{k}\right) \Phi_{m} t^{m},
$$

where inversion is defined in the sense of formal power series. 
Proof. From (1.1), we have

$$
g(t)=\sum_{n=0}^{\infty} \Phi_{n} t^{n}=\sum_{m=0}^{N-1} \Phi_{m} t^{m}+G(t)
$$

where

$$
G(t) \equiv \sum_{n=N}^{\infty} \Phi_{n} t^{n}=\sum_{n=N}^{\infty}\left[\sum_{k=0}^{N-1} a_{k} \Phi_{n-N+k}\right] t^{n} .
$$

With $n-N+k=m$, this is rewritten as

$$
G(t)=\sum_{k=0}^{N-1} a_{k} t^{N-k} \sum_{m=k}^{\infty} \Phi_{m} t^{m}=\sum_{k=0}^{N-1} a_{k} t^{N-k}\left[g(t)-\sum_{m=0}^{k-1} \Phi_{m} t^{m}\right] .
$$

With $N-k=j$, it can be further rewritten as

$$
G(t)=\sum_{j=1}^{N} a_{N-j} t^{j}\left[g(t)-\sum_{m=0}^{N-j-1} \Phi_{m} t^{m}\right]
$$

that is,

$$
G(t)=\left[\sum_{j=1}^{N} a_{N-j} t^{j}\right] g(t)-\sum_{m=0}^{N-2}\left[\sum_{j=1}^{N-m-1} a_{N-j} t^{j}\right] \Phi_{m} t^{m} .
$$

Substituting this expression into (2.4), we find

$$
\left[1-\sum_{k=1}^{N} a_{N-k} t^{k}\right] g(t)=\sum_{m=0}^{N-1} \Phi_{m} t^{m}-\sum_{m=0}^{N-2}\left[\sum_{j=1}^{N-m-1} a_{N-j} t^{j}\right] \Phi_{m} t^{m} .
$$

Although the summation over $m$ ends at $N-2$ in the second term of the righthand side, it can be extended to $N-1$ without bringing any change because the summation over $j$ is absent for $m=N-1$. Finally, multiplying the above expression by $\left(1-\sum_{k=1}^{N} a_{N-k} t^{k}\right)^{-1}$ from the left, we obtain (2.3).

The following equation for $\alpha$ is called the characteristic equation of the recurrence relation:

$$
\alpha^{N}-\sum_{k=0}^{N-1} a_{k} \alpha^{k}=0 .
$$

Although the fundamental theorem of algebra no longer holds when the coefficients are non-commutative quantities, we assume that (2.5) has $N$ solutions $\alpha_{1}, \ldots, \alpha_{N}$, and for simplicity that they are all different (if some of them are equal, we treat them as the limiting case). 
Proposition 2.2. The exponential generating function (2.2) is given by

$$
f(t)=\sum_{j=1}^{N} e^{\alpha_{j} t} C_{j}
$$

where $\left\{C_{1}, \ldots, C_{N}\right\}$ is the solution to the simultaneous linear equations

$$
\sum_{j=1}^{N} \alpha_{j}^{m} C_{j}=\Phi_{m} \quad(m=0,1, \ldots, N-1) .
$$

Proof. Because $\left.\left(\frac{d}{d t}\right)^{n} f(t)\right|_{t=0}=\Phi_{n}$, (1.1) implies that $f(t)$ satisfies the linear differential equation

$$
\left[\left(\frac{d}{d t}\right)^{N}-\sum_{k=0}^{N-1} a_{k}\left(\frac{d}{d t}\right)^{k}\right] f(t)=0
$$

Solving this equation, we obtain (2.6) with (2.7).

Since

$$
\int_{0}^{\infty} d t e^{-p t} \frac{t^{n}}{n !}=p^{-n-1}
$$

we find that the Laplace transform of $f(t)$ is essentially given by $g(t)$, that is,

$$
\int_{0}^{\infty} d t e^{-p t} f(t)=\int_{0}^{\infty} d t \sum_{j=1}^{N} e^{-\left(p-\alpha_{j}\right) t} C_{j}=\sum_{j=1}^{N}\left(p-\alpha_{j}\right)^{-1} C_{j}
$$

should coincide with $p^{-1} g\left(p^{-1}\right)$. Hence, setting $p^{-1}=t$, we should have the following theorem.

Theorem 2.1. Let $\alpha_{1}, \ldots, \alpha_{N}$ be $N$ mutually different solutions to the algebraic equation

$$
\alpha^{N}-\sum_{k=0}^{N-1} a_{k} \alpha^{k}=0
$$

where the coefficients $a_{0}, a_{1}, \ldots, a_{N-1}$ are mutually generically non-commutative. Furthermore, let $C_{j}$ be quantities generically non-commutative with the $a_{k}$, and $t$ be a commutative parameter. Then the following identity holds:

$$
\sum_{j=1}^{N}\left(1-\alpha_{j} t\right)^{-1} C_{j}=g(t),
$$


where

$$
g(t)=\left(1-\sum_{k=1}^{N} a_{N-k} t^{k}\right)^{-1} \sum_{m=0}^{N-1}\left(1-\sum_{k=1}^{N-m-1} a_{N-k} t^{k}\right) t^{m} \sum_{j=1}^{N} \alpha_{j}^{m} C_{j} .
$$

Here inversion is defined in the sense of formal power series.

Since both (2.1) and (2.2) are formal series, the reasoning based on the Laplace transform cannot be regarded as a proof. The direct proof of the theorem is presented in Section 6 (of course, without assuming the commutativity between $\alpha_{j}$ 's).

\section{$\S 3$. The case of $N=2$}

To begin with, we consider the case of $N=2$, in which (2.3) with (2.7) reduces to

$$
g(t)=\left(1-a_{1} t-a_{0} t^{2}\right)^{-1}\left[\left(1-a_{1} t+\alpha_{1} t\right) C_{1}+\left(1-a_{1} t+\alpha_{2} t\right) C_{2}\right],
$$

where

$$
\alpha_{1}^{2}-a_{1} \alpha_{1}-a_{0}=0, \quad \alpha_{2}^{2}-a_{1} \alpha_{2}-a_{0}=0 .
$$

The characteristic equation is the quadratic equation $\alpha^{2}-a_{1} \alpha-a_{0}=0$. We investigate relations between its solutions and its coefficients without assuming commutativity. First, from the difference between the two equations of (3.2), we find

$$
\alpha_{1}^{2}-\alpha_{2}^{2}-a_{1}\left(\alpha_{1}-\alpha_{2}\right)=0
$$

Therefore,

$$
a_{1}=\left(\alpha_{1}^{2}-\alpha_{2}^{2}\right)\left(\alpha_{1}-\alpha_{2}\right)^{-1},
$$

where we assume that $\alpha_{1}-\alpha_{2}$ is invertible.

Substitution of this result into the first equation of (3.2) yields

$$
\begin{aligned}
a_{0} & =\alpha_{1}^{2}-\left(\alpha_{1}^{2}-\alpha_{2}^{2}\right)\left(\alpha_{1}-\alpha_{2}\right)^{-1} \alpha_{1} \\
& =\alpha_{1}^{2}\left(\alpha_{2}-\alpha_{1}\right)^{-1} \alpha_{2}+\alpha_{2}^{2}\left(\alpha_{1}-\alpha_{2}\right)^{-1} \alpha_{1} .
\end{aligned}
$$

Substituting these expressions for $a_{1}$ and $a_{0}$ into $g(t)$, we have

$$
\begin{aligned}
g(t)= & {[\psi(t)]^{-1}\left[\left\{1+\alpha_{1} t-\left(\alpha_{1}^{2}-\alpha_{2}^{2}\right)\left(\alpha_{1}-\alpha_{2}\right)^{-1} t\right\} C_{1}\right.} \\
& \left.+\left\{1+\alpha_{2} t-\left(\alpha_{2}^{2}-\alpha_{1}^{2}\right)\left(\alpha_{2}-\alpha_{1}\right)^{-1} t\right\} C_{2}\right],
\end{aligned}
$$

where

$$
\begin{aligned}
\psi(t) \equiv & 1-\left(\alpha_{1}^{2}-\alpha_{2}^{2}\right)\left(\alpha_{1}-\alpha_{2}\right)^{-1} t \\
& -\left\{\alpha_{1}^{2}\left(\alpha_{2}-\alpha_{1}\right)^{-1} \alpha_{2}+\alpha_{2}^{2}\left(\alpha_{1}-\alpha_{2}\right)^{-1} \alpha_{1}\right\} t^{2} .
\end{aligned}
$$


The fact that (3.3) with (3.4) is the right answer can be confirmed by rewriting $\psi(t)$ in the following way:

$$
\begin{aligned}
\psi(t) & =1+\alpha_{1}^{2}\left(\alpha_{2}-\alpha_{1}\right)^{-1}\left(1-\alpha_{2} t\right) t+\alpha_{2}^{2}\left(\alpha_{1}-\alpha_{2}\right)^{-1}\left(1-\alpha_{1} t\right) t \\
& =1-\alpha_{1}^{2} t^{2}+\alpha_{1}^{2}\left(\alpha_{2}-\alpha_{1}\right)^{-1}\left(1-\alpha_{1} t\right) t+\alpha_{2}^{2}\left(\alpha_{1}-\alpha_{2}\right)^{-1}\left(1-\alpha_{1} t\right) t \\
& =\left[1+\alpha_{1} t-\left(\alpha_{1}^{2}-\alpha_{2}^{2}\right)\left(\alpha_{1}-\alpha_{2}\right)^{-1} t\right]\left(1-\alpha_{1} t\right) ;
\end{aligned}
$$

that is, the factor $1-\alpha_{1} t$ has been moved to the right. Since $(A B)^{-1}=B^{-1} A^{-1}$, the left factor cancels with the coefficient of $C_{1}$ in the numerator of (3.1). Likewise, the factor $1-\alpha_{2} t$ can be taken out from $\psi(t)$.

Thus, it is interesting to note that $\psi(t)$ can be factorized in two different ways, though, of course, both factorizations reduce to $\left(1-\alpha_{1} t\right)\left(1-\alpha_{2} t\right)$ in the commutative case.

Remark. In general, factorizations are not necessarily unique in the polynomial whose coefficients are non-commutative quantities.

\section{$\S 4$. Relations between solutions and coefficients}

In order to express the denominator function,

$$
\psi(t)=1-\sum_{k=1}^{N} a_{N-k} t^{k},
$$

of $g(t)$ in terms of $\alpha_{1}, \ldots, \alpha_{N}$, we need the relation between the solutions to an algebraic equation and the coefficients appearing in it in the non-commutative case. This problem was discussed in the theory of quasi-determinants developed by Gelfand et al. [2]. We here consider this problem without referring to quasideterminants explicitly.

Even in an ordinary matrix, if it is decomposed into some blocks, those blocks may be regarded as non-commutative elements. Below we make use of the wellknown inversion formula for a matrix in block form.

Proposition 4.1. Let $A, B, C, D$ be generically non-commutative quantities. The inverse of the nonsingular matrix

$$
M=\left[\begin{array}{ll}
A & B \\
C & D
\end{array}\right]
$$

is given by

$$
M^{-1}=\left[\begin{array}{ll}
\left(A-B D^{-1} C\right)^{-1} & \left(C-D B^{-1} A\right)^{-1} \\
\left(B-A C^{-1} D\right)^{-1} & \left(D-C A^{-1} B\right)^{-1}
\end{array}\right],
$$

provided that $A, B, C, D$ are invertible. 
Proof. We have only to confirm that the product of both matrices becomes the unit matrix. This is easily seen from

$$
\begin{aligned}
& \left(B-A C^{-1} D\right)^{-1}=\left[\left(B D^{-1} C-A\right) C^{-1} D\right]^{-1}=-D^{-1} C\left(A-B D^{-1} C\right)^{-1}, \\
& \left(C-D B^{-1} A\right)^{-1}=\left[\left(C A^{-1} B-D\right) B^{-1} A\right]^{-1}=-A^{-1} B\left(D-C A^{-1} B\right)^{-1} .
\end{aligned}
$$

Since the existence of $M^{-1}$ is assumed, the above formal expression must be the correct expression for $M^{-1}$.

In order to calculate the determinant of the inverse matrix in the general case, we need not extend this formula to the form of $N \times N$ blocks, because we can regard $A, B, C, D$ as blocks consisting of non-commutative elements.

Now, we wish to solve the simultaneous equations for $a_{N-1}, a_{N-2}, \ldots, a_{0}$,

$$
\alpha_{j}^{N}-a_{N-1} \alpha_{j}^{N-1}-\cdots-a_{1} \alpha_{j}-a_{0}=0 \quad(j=1, \ldots, N) .
$$

We set

$$
V\left(\alpha_{1}, \ldots, \alpha_{N}\right) \equiv\left[\begin{array}{cccc}
\alpha_{1}^{N-1} & \alpha_{2}^{N-1} & \ldots & \alpha_{N}^{N-1} \\
\alpha_{1}^{N-2} & \alpha_{2}^{N-2} & \ldots & \alpha_{N}^{N-2} \\
\ldots \ldots & \ldots \ldots \ldots \ldots . . \\
\alpha_{1} & \alpha_{2} & \ldots & \alpha_{N} \\
1 & 1 & \ldots & 1
\end{array}\right]
$$

then (4.1) is rewritten in matrix form as

$$
\left[\begin{array}{llll}
a_{N-1} & a_{N-2} & \ldots & a_{0}
\end{array}\right] V\left(\alpha_{1}, \ldots, \alpha_{N}\right)=\left[\begin{array}{lll}
\alpha_{1}^{N} & \ldots & \alpha_{N}^{N}
\end{array}\right]
$$

Hence $a_{N-1}, a_{N-2}, \ldots, a_{0}$ are expressed as

$$
\left[\begin{array}{llll}
a_{N-1} & a_{N-2} & \ldots & a_{0}
\end{array}\right]=\left[\begin{array}{lll}
\alpha_{1}^{N} & \cdots & \alpha_{N}^{N}
\end{array}\right]\left[V\left(\alpha_{1}, \ldots, \alpha_{N}\right)\right]^{-1} .
$$

Thus our problem is to calculate the inverse matrix of $V\left(\alpha_{1}, \ldots, \alpha_{N}\right)$.

For example, in the case of $N=2$, since

$$
V\left(\alpha_{1}, \alpha_{2}\right)=\left[\begin{array}{cc}
\alpha_{1} & \alpha_{2} \\
1 & 1
\end{array}\right]
$$

Proposition 4.1 implies

$$
\left[V\left(\alpha_{1}, \alpha_{2}\right)\right]^{-1}=\left[\begin{array}{cc}
\left(\alpha_{1}-\alpha_{2}\right)^{-1} & \left(\alpha_{2}-\alpha_{1}\right)^{-1} \alpha_{2} \\
\left(\alpha_{2}-\alpha_{1}\right)^{-1} & \left(\alpha_{1}-\alpha_{2}\right)^{-1} \alpha_{1}
\end{array}\right]
$$

Therefore, we have

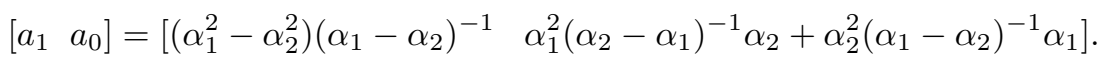

Of course, this result coincides with the one given in Section 3. 


\section{$\S 5$. The case of $N=3$}

It is quite nontrivial already in the case of $N=3$ to express $a_{0}, \ldots, a_{N-1}$ in terms of $\alpha_{j}$ 's.

\section{$\S 5.1$. Calculation of $a_{2}$}

First, we rewrite the matrix

$$
V\left(\alpha_{1}, \alpha_{2}, \alpha_{3}\right)=\left[\begin{array}{ccc}
\alpha_{1}^{2} & \alpha_{2}^{2} & \alpha_{3}^{2} \\
\alpha_{1} & \alpha_{2} & \alpha_{3} \\
1 & 1 & 1
\end{array}\right]
$$

in the following block form:

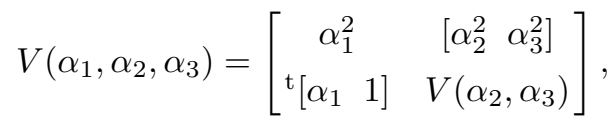

where $\mathrm{t}$ denotes transposition. By using Proposition 4.1, we calculate the $(1,1)$ element of $\left[V\left(\alpha_{1}, \alpha_{2}, \alpha_{3}\right)\right]^{-1}$. Since

$$
\left[V\left(\alpha_{2}, \alpha_{3}\right)\right]^{-1}=\left[\begin{array}{c}
\left(\alpha_{2}-\alpha_{3}\right)^{-1}\left(\alpha_{3}-\alpha_{2}\right)^{-1} \alpha_{3} \\
\left(\alpha_{3}-\alpha_{2}\right)^{-1}\left(\alpha_{2}-\alpha_{3}\right)^{-1} \alpha_{2}
\end{array}\right]
$$

we find

$$
\begin{aligned}
{\left[\left[V\left(\alpha_{1}, \alpha_{2}, \alpha_{3}\right)\right]^{-1}\right]_{11}=\left\{\alpha_{1}^{2}-\left[\alpha_{2}^{2} \alpha_{3}^{2}\right]\left[V\left(\alpha_{2}, \alpha_{3}\right)\right]^{-1 \mathrm{t}}\left[\begin{array}{ll}
\alpha_{1} & 1
\end{array}\right]\right\}^{-1} } \\
\quad=\left\{\alpha_{1}^{2}-\alpha_{2}^{2}\left(\alpha_{2}-\alpha_{3}\right)^{-1}\left(\alpha_{1}-\alpha_{3}\right)-\alpha_{3}^{2}\left(\alpha_{3}-\alpha_{2}\right)^{-1}\left(\alpha_{1}-\alpha_{2}\right)\right\}^{-1} .
\end{aligned}
$$

The $(2,1)$-element and the $(3,1)$-element are obtained by cyclic permutations of subscripts $(1 \rightarrow 2 \rightarrow 3 \rightarrow 1)$. Thus,

$$
a_{2}=\sum_{i, j, k: \text { cycl. }} \alpha_{i}^{3}\left\{\alpha_{i}^{2}-\alpha_{j}^{2}\left(\alpha_{j}-\alpha_{k}\right)^{-1}\left(\alpha_{i}-\alpha_{k}\right)-\alpha_{k}^{2}\left(\alpha_{k}-\alpha_{j}\right)^{-1}\left(\alpha_{i}-\alpha_{j}\right)\right\}^{-1} .
$$

\section{§5.2. Calculation of $a_{1}$}

In this case, in order to apply Proposition 4.1, we move the top row of the matrix $V$ to the bottom. Setting

$$
V^{\prime}\left(\alpha_{2}, \alpha_{3}\right)=\left[\begin{array}{cc}
1 & 1 \\
\alpha_{2}^{2} & \alpha_{3}^{2}
\end{array}\right]
$$

we have

$$
\left[V^{\prime}\left(\alpha_{2}, \alpha_{3}\right)\right]^{-1}=\left[\begin{array}{cc}
\left(\alpha_{3}^{2}-\alpha_{2}^{2}\right)^{-1} \alpha_{3}^{2} & \left(\alpha_{2}^{2}-\alpha_{3}^{2}\right)^{-1} \\
\left(\alpha_{2}^{2}-\alpha_{3}^{2}\right)^{-1} \alpha_{2}^{2} & \left(\alpha_{3}^{2}-\alpha_{2}^{2}\right)^{-1}
\end{array}\right]
$$


Applying Proposition 4.1 to the modified form of $V$, we obtain

$$
\begin{aligned}
{\left[\left[V\left(\alpha_{1}, \alpha_{2}, \alpha_{3}\right)\right]^{-1}\right]_{12}=\left\{\alpha_{1}-\left[\begin{array}{ll}
\alpha_{2} & \alpha_{3}
\end{array}\right]\left[V^{\prime}\left(\alpha_{2}, \alpha_{3}\right)\right]^{-1}{ }^{\mathrm{t}}\left[\begin{array}{ll}
1 & \alpha_{1}^{2}
\end{array}\right]\right\}^{-1} } \\
=\left\{\alpha_{1}-\alpha_{2}\left(\alpha_{2}^{2}-\alpha_{3}^{2}\right)^{-1}\left(\alpha_{1}^{2}-\alpha_{3}^{2}\right)-\alpha_{3}\left(\alpha_{3}^{2}-\alpha_{2}^{2}\right)^{-1}\left(\alpha_{1}^{2}-\alpha_{2}^{2}\right)\right\}^{-1} .
\end{aligned}
$$

Thus,

$$
a_{1}=\sum_{i, j, k: \text { cycl. }} \alpha_{i}^{3}\left\{\alpha_{i}-\alpha_{j}\left(\alpha_{j}^{2}-\alpha_{k}^{2}\right)^{-1}\left(\alpha_{i}^{2}-\alpha_{k}^{2}\right)-\alpha_{k}\left(\alpha_{k}^{2}-\alpha_{j}^{2}\right)^{-1}\left(\alpha_{i}^{2}-\alpha_{j}^{2}\right)\right\}^{-1} .
$$

\section{$\S 5.3$. Calculation of $a_{0}$}

We move the bottom row of $V$ to the top. Setting

$$
V^{\prime \prime}\left(\alpha_{2}, \alpha_{3}\right)=\left[\begin{array}{ll}
\alpha_{2}^{2} & \alpha_{3}^{2} \\
\alpha_{2} & \alpha_{3}
\end{array}\right]
$$

we have

$$
\left[V^{\prime \prime}\left(\alpha_{2}, \alpha_{3}\right)\right]^{-1}=\left[\begin{array}{cc}
\alpha_{2}^{-1}\left(\alpha_{2}-\alpha_{3}\right)^{-1} & \alpha_{2}^{-1}\left(\alpha_{3}-\alpha_{2}\right)^{-1} \alpha_{3} \\
\alpha_{3}^{-1}\left(\alpha_{3}-\alpha_{2}\right)^{-1} & \alpha_{3}^{-1}\left(\alpha_{2}-\alpha_{3}\right)^{-1} \alpha_{2}
\end{array}\right]
$$

Hence, Proposition 4.1 implies

$$
\begin{aligned}
& {\left[\left[V\left(\alpha_{1}, \alpha_{2}, \alpha_{3}\right)\right]^{-1}\right]_{13}=\left\{1-\left[\begin{array}{ll}
1 & 1
\end{array}\right]\left[V^{\prime \prime}\left(\alpha_{2}, \alpha_{3}\right)\right]^{-1}{ }^{\mathrm{t}}\left[\begin{array}{ll}
\alpha_{1}^{2} & \alpha_{1}
\end{array}\right]\right\}^{-1}} \\
& \quad=\left\{1-\alpha_{2}^{-1}\left(\alpha_{2}-\alpha_{3}\right)^{-1}\left(\alpha_{1}-\alpha_{3}\right) \alpha_{1}-\alpha_{3}^{-1}\left(\alpha_{3}-\alpha_{2}\right)^{-1}\left(\alpha_{1}-\alpha_{2}\right) \alpha_{1}\right\}^{-1} .
\end{aligned}
$$

Thus,

$$
\begin{aligned}
a_{0}=\sum_{i, j, k: \text { cycl. }} \alpha_{i}^{3}\left\{1-\alpha_{j}^{-1}\left(\alpha_{j}-\alpha_{k}\right)^{-1}(\right. & \left.\alpha_{i}-\alpha_{k}\right) \alpha_{i} \\
& \left.-\alpha_{k}^{-1}\left(\alpha_{k}-\alpha_{j}\right)^{-1}\left(\alpha_{i}-\alpha_{j}\right) \alpha_{i}\right\}^{-1} .
\end{aligned}
$$

\section{§5.4. Reduction in the commutative case}

If $\alpha_{1}, \alpha_{2}$ and $\alpha_{3}$ are mutually commutative, (5.1), (5.2) and (5.3) reduce to

$$
\begin{aligned}
& a_{2}^{\text {comm }}=\frac{\alpha_{1}^{3}\left(\alpha_{2}-\alpha_{3}\right)+\alpha_{2}^{3}\left(\alpha_{3}-\alpha_{1}\right)+\alpha_{3}^{3}\left(\alpha_{1}-\alpha_{2}\right)}{\alpha_{1}^{2}\left(\alpha_{2}-\alpha_{3}\right)+\alpha_{2}^{2}\left(\alpha_{3}-\alpha_{1}\right)+\alpha_{3}^{2}\left(\alpha_{1}-\alpha_{2}\right)}=\alpha_{1}+\alpha_{2}+\alpha_{3}, \\
& a_{1}^{\text {comm }}=\frac{\alpha_{1}^{3}\left(\alpha_{2}^{2}-\alpha_{3}^{2}\right)+\alpha_{2}^{3}\left(\alpha_{3}^{2}-\alpha_{1}^{2}\right)+\alpha_{3}^{3}\left(\alpha_{1}^{2}-\alpha_{2}^{2}\right)}{\alpha_{1}\left(\alpha_{2}^{2}-\alpha_{3}^{2}\right)+\alpha_{2}\left(\alpha_{3}^{2}-\alpha_{1}^{2}\right)+\alpha_{3}\left(\alpha_{1}^{2}-\alpha_{2}^{2}\right)}=-\left(\alpha_{1} \alpha_{2}+\alpha_{2} \alpha_{3}+\alpha_{3} \alpha_{1}\right), \\
& a_{0}^{\text {comm }}=\frac{\alpha_{1}^{3} \alpha_{2} \alpha_{3}\left(\alpha_{2}-\alpha_{3}\right)+\alpha_{2}^{3} \alpha_{3} \alpha_{1}\left(\alpha_{3}-\alpha_{1}\right)+\alpha_{3}^{3} \alpha_{1} \alpha_{2}\left(\alpha_{1}-\alpha_{2}\right)}{\alpha_{2} \alpha_{3}\left(\alpha_{2}-\alpha_{3}\right)+\alpha_{3} \alpha_{1}\left(\alpha_{3}-\alpha_{1}\right)+\alpha_{1} \alpha_{2}\left(\alpha_{1}-\alpha_{2}\right)}=\alpha_{1} \alpha_{2} \alpha_{3},
\end{aligned}
$$

respectively. That is, $a_{2},-a_{1}$ and $a_{0}$ reproduce the fundamental symmetric functions of $\alpha_{j}$ 's, as it should be. 


\section{§6. Proof of Theorem 2.1}

For the general $N$, it is quite laborious to write down the concrete expressions for $a_{0}, a_{1}, \ldots, a_{N-1}$ in terms of $\alpha_{j}$ 's, but it is certainly possible, as shown in Section 4 . We now prove Theorem 2.1, that is, we show that the identity (2.9) with (2.10) indeed holds.

Proof. Multiplying (2.9) with (2.10) by $1-\sum_{k=1}^{N} a_{N-k} t^{k}$ from the left, and comparing the coefficients of $C_{j}$ on both sides, we see that the formula to be proved is

$$
\left(1-\sum_{k=1}^{N} a_{N-k} t^{k}\right)\left(1-\alpha_{j} t\right)^{-1}=\sum_{m=0}^{N-1}\left(1-\sum_{k=1}^{N-m-1} a_{N-k} t^{k}\right) \alpha_{j}^{m} t^{m} .
$$

Furthermore, multiplying this formula by $1-\alpha_{j} t$ from the right, we have

$$
\left[\sum_{m=0}^{N-1}\left(1-\sum_{k=1}^{N-m-1} a_{N-k} t^{k}\right) \alpha_{j}^{m} t^{m}\right]\left(1-\alpha_{j} t\right)=1-\sum_{k=1}^{N} a_{N-k} t^{k} .
$$

In the following we prove this identity.

We denote the left-hand side of (6.2) by $F(t)$. Setting $m+k=n$ in $F(t)$, we have

$$
F(t)=\left[\sum_{n=0}^{N-1}\left(\alpha_{j}^{n}-\sum_{k=1}^{n} a_{N-k} \alpha_{j}^{n-k}\right) t^{n}\right]\left(1-\alpha_{j} t\right) .
$$

Rewriting this expression in powers of $t$, we obtain

$$
\begin{aligned}
F(t)= & +\sum_{n=1}^{N-1}\left[\left(\alpha_{j}^{n}-\sum_{k=1}^{n} a_{N-k} \alpha_{j}^{n-k}\right)-\alpha_{j}\left(\alpha_{j}^{n-1}-\sum_{k=1}^{n-1} a_{N-k} \alpha_{j}^{n-k-1}\right)\right] t^{n} \\
& -\alpha_{j}\left(\alpha_{j}^{N-1}-\sum_{k=1}^{N-1} a_{N-k} \alpha_{j}^{N-k-1}\right) t^{N} .
\end{aligned}
$$

The expression in square brackets cancels except for the term $k=n$. Then we find

$$
F(t)=1-\sum_{n=1}^{N-1} a_{N-n} t^{n}-\left(\alpha_{j}^{N}-\sum_{k=1}^{N-1} a_{N-k} \alpha_{j}^{N-k}\right) t^{N} .
$$

Since $\alpha_{j}$ is a solution to $(2.8)$, we see that

$$
\alpha_{j}^{N}-\sum_{k=1}^{N-1} a_{N-k} \alpha_{j}^{N-k}=a_{0} .
$$


Hence, we obtain

$$
F(t)=1-\sum_{n=1}^{N-1} a_{N-n} t^{n}-a_{0} t^{N}=1-\sum_{n=1}^{N} a_{N-n} t^{n},
$$

which coincides with the right-hand side of (6.2).

\section{$\S 7$. Discussion}

In the present paper, we have discussed a homogeneous linear recurrence sequence with non-commutative coefficients. Its ordinary generating function, $g(t)$, and its exponential generating function, $f(t)$, are defined and their relation is explicitly established.

Our main theorem can be regarded as the reduction to a common denominator for a sum of fractions of generically non-commutative quantities. Indeed, (2.9) with (2.10) can be written as

$$
\sum_{j=1}^{N}\left(1-\alpha_{j} t\right)^{-1} C_{j}=[\psi(t)]^{-1} \sum_{j=1}^{N} \varphi_{j}(t) C_{j},
$$

where $\psi(t)$ is totally symmetric, $\sum_{j} \varphi_{j}(t) C_{j}$ being manifestly invariant under simultaneous permutations of $\left\{\alpha_{1}, \ldots, \alpha_{N}\right\}$ and $\left\{C_{1}, \ldots, C_{N}\right\}$, and none of them involves $\alpha_{j}^{-1}$. Setting $A_{j}=1-\alpha_{j} t{ }^{1}$ we may restate this result in the following way:

Corollary 7.1. Let $A_{1}, \ldots, A_{N} ; B_{1}, \ldots, B_{N} ; C_{1}, \ldots, C_{N}$ be generically non-commutative quantities. Then the following formal identity holds:

$$
\sum_{j=1}^{N} B_{j} A_{j}^{-1} C_{j}=\sum_{j=1}^{N} B_{j}[\psi(A)]^{-1} \varphi_{j}(A) C_{j},
$$

where $\psi(A)$ and $\varphi_{j}(A)$ 's are functions of $A_{1}, \ldots, A_{N}$ involving no $A_{k}^{-1}$, and $\psi(A)$ is totally symmetric, $\sum_{j=1}^{N} B_{j} \varphi_{j}(A) C_{j}$ being manifestly invariant under simultaneous permutations of $\left\{A_{1}, \ldots, A_{N}\right\},\left\{B_{1}, \ldots, B_{N}\right\}$ and $\left\{C_{1}, \ldots, C_{N}\right\}$.

For example, in the case of $N=2$, the concrete expressions are as follows:

$$
\begin{aligned}
B_{1} A_{1}^{-1} C_{1} & +B_{2} A_{2}^{-1} C_{2} \\
& =B_{1}\left[\psi\left(A_{1}, A_{2}\right)\right]^{-1} \varphi_{1}\left(A_{1}, A_{2}\right) C_{1}+B_{2}\left[\psi\left(A_{1}, A_{2}\right)\right]^{-1} \varphi_{2}\left(A_{1}, A_{2}\right) C_{2},
\end{aligned}
$$

\footnotetext{
${ }^{1}$ Then, of course, the formal power series expansion with respect to $t$ becomes impossible.
} 
where

$$
\begin{aligned}
\psi\left(A_{1}, A_{2}\right) & =A_{1}^{2}\left(A_{1}-A_{2}\right)^{-1} A_{2}+A_{2}^{2}\left(A_{2}-A_{1}\right)^{-1} A_{1}, \\
\varphi_{1}\left(A_{1}, A_{2}\right) & =-A_{1}+\left(A_{1}^{2}-A_{2}^{2}\right)\left(A_{1}-A_{2}\right)^{-1}, \\
\varphi_{2}\left(A_{1}, A_{2}\right) & =-A_{2}+\left(A_{2}^{2}-A_{1}^{2}\right)\left(A_{2}-A_{1}\right)^{-1},
\end{aligned}
$$

as is derived from the results given in Section 3. Of course, if $A_{1}$ and $A_{2}$ commute, the expressions reduce to $\psi^{\text {comm }}=A_{1} A_{2}, \varphi_{1}^{\text {comm }}=A_{2}, \varphi_{2}^{\text {comm }}=A_{1}$.

\section{References}

[1] P. M. Batchelder, An introduction to linear difference equations, Dover Publ., 1967. MR 0216187

[2] I. Gelfand, D. Krob, A. Lascoux, B. Leclerc, V. Retakh, and J. Thibon, Noncommutative symmetric functions, Adv. Math. 112 (1995), 218-348. Z Zbl 0831.05063 MR 1327096

[3] S. K. Lando, Lectures on generating functions, Amer. Math. Soc., 2003. Zbl 1032.05001 MR 2013270

[4] K. S. Miller, Linear difference equations, W. A. Benjamin, 1968. Zbl 0162.40201 MR 0227644 